\title{
De Novo Dural Arteriovenous Fistula Formation Following Asymptomatic Sinus Thrombosis: A Case Report and Review of the Literature
}

\author{
Lei Li ${ }^{a}$, Darryn Shaff ${ }^{\mathrm{a}, \mathrm{b}, \mathrm{c}}$, Yuebing $\mathrm{Li}^{\mathrm{a}}$
}

\begin{abstract}
Cerebral venous sinus thrombosis (CVST) has been suggested to play an important role in the pathogenesis of dural arteriovenous fistular (DAVF) formation but studies supporting a causal relationship are rare. We report a 15 -year-old male who developed a brief syncopal episode that was felt to be secondary to dehydration. A non-contrast computerized tomography (CT) scan of the head showed hyperdensities of the posterior superior sagittal and bilateral transverse sinuses. Magnetic resonance venogram confirmed the presence of diffuse CVST without cerebral edema, infarction or hemorrhage. Dural sinuses were partially recanalized after a sixweek anticoagulation treatment. At 14 months patient was found with a left sided DAVF that was embolized successfully without complications. The presence of asymptomatic, minimally symptomatic or partially treated CVST may be implicated in the pathogenesis of DAVF.
\end{abstract}

Keywords: Dural arteriovenous fistula; Arteriovenous malformation; Cerebral venous sinus thrombosis; Stroke

\section{Introduction}

Dural arteriovenous fistulas (DAVFs) are abnormal intracranial vascular channels through which meningeal or extracranial arteries shunt blood directly into mostly dural sinuses, and rarely meningeal or subarachnoid veins. DAVFs are usually located at the dura mater within or near a dural sinus wall. Cerebral venous sinus thrombosis (CVST) has been suggested to play an important role in the pathogenesis of

Manuscript accepted for publication April 23, 2013

a Division of Neurology, Department of Medicine, Lehigh Valley Health Network, Allentown, Pennsylvania, USA

${ }^{\mathrm{b}}$ Division of Neurointerventional Radiology, Department of Radiology,

Lehigh Valley Health Network, Allentown, Pennsylvania, USA

${ }^{\mathrm{c}}$ Corresponding author: Darryn Shaff, Neuroscience Service Line, 1210

S. Cedar Crest Blvd., Suite 1200, Allentown, PA 18103, USA.

Email: Darryn.Shaff@lvhn.org

doi: http://dx.doi.org/10.4021/jnr195w
DAVF but studies supporting a causal relationship are rare [1]. Here we report a rare case of de novo DAVF formation following the occurrence of extensive but asymptomatic CVSTs.

\section{Case Report}

A 15-year-old Asian male developed a brief syncopal episode the day after taking a long international flight trip and suffering dehydration from prolonged poor oral intake. There were no other symptoms. His neurological examination was entirely normal. A non-contrast computerized tomography (CT) scan of the head showed abnormal hyperdensities of the posterior superior sagittal and bilateral transverse sinuses (Fig. 1). Magnetic resonance venogram (MRV) and magnetic resonance imaging (MRI) of the head confirmed the presence of diffuse venous thrombosis in the superior sagittal, straight, bilateral transverse, and left sigmoid sinuses without the presence of parenchymal edema, infarction or hemorrhage (Fig. 1). Hypercoagulable studies including antiphospholipid antibody, fibrinogen, antithrombin III, plasminogen and activated protein $\mathrm{C}$ resistance were all within normal limits. Factor V Leiden and prothrombin G20210A mutational analyses were negative. Intravenous heparin treatment was initiated, followed by oral warfarin with a target International Normalized Ratio (INR) of 2.0-3.0. At 6 weeks, a repeated MRV showed a marked improvement with prominent venous flow seen in the superior sagittal and right transverse sinuses. The left transverse sinus recanalized but remained narrow, and both the left sigmoid sinus and jugular vein were patent. The brain parenchyma remained entirely normal on MRI (Fig. 1). The warfarin therapy was discontinued.

At 14 months, the patient started to experience left occipital and upper neck pain upon strenuous exercises. Papilledema was noted on the funduscopic examination but the remaining neurological exam was normal. Head MRV showed a lack of significant flow in the left sigmoid sinus and jugular vein but otherwise remained the same when compared to the previous study. MRI of the brain did not show any infarction or hemorrhage but revealed new findings of abnormally 


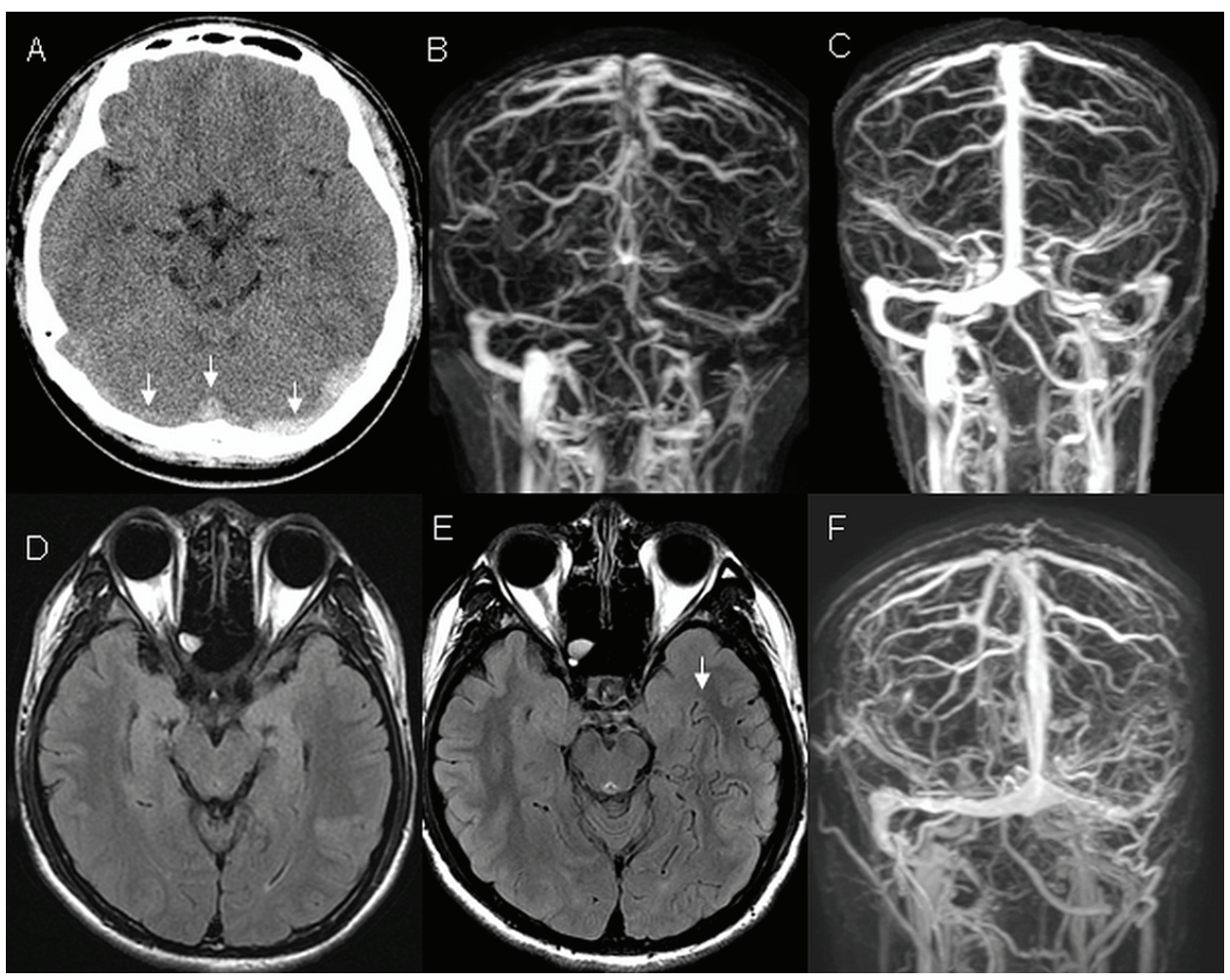

Figure 1. Head imaging. At initial presentation, non-contrast computerized tomography (CT) of the head (A) demonstrated hyperdensities in multiple sinuses including bilateral transverse and superior sagittal sinuses (arrowheads) which presence was verified on magnetic resonance venogram (MRV) (B). After a six-week anticoagulation treatment, repeated MRV (C) showed prominent flow in the superior sagittal and right transverse sinuses. The left transverse sinus recanalized but remained narrow. Flow was also seen in the left sigmoid sinus and left jugular vein. Fluid attenuated inversion recovery (FLAIR) image at six weeks (D) was normal but FLAIR image at 14 months (E) revealed abnormally dilated cerebral vessels representing venous congestion (arrowhead). Follow up MRV at 14 months (F) demonstrated an occlusion of left sigmoid sinus and left jugular vein.

dilated cerebral vessels representing venous congestion in the left sided supratentorial regions (Fig. 1). Cerebral angiogram confirmed the presence of a left transverse-sigmoid DAVF with ipsilateral transverse-sigmoid sinus occlusion and prominent cortical venous reflux (Fig. 2). At 16 months the patient underwent endovascular embolization of multiple feeding branches arising from internal carotid, external carotid and vertebral distributions, using a mixture of n-butyl cyanoacrylate and ethiodized oil prepared from the TRUFILL liquid embolic system (Codman neurovascular, Codman \& Shurtleff, Inc., Raynham, Massachusetts, USA). Follow-up cerebral angiograms at 19 and 31 months showed a completely resolved DAVF. At 32 month follow-up, patient remained free of symptoms with a completely normal neurological examination.

\section{Discussion}

Intracranial DAVF accounts for $10-15 \%$ of all intracranial vascular malformations. While the earlier literature speculat- ed that DAVFs were congenital lesions, recent data suggested that they are probably acquired. DAVFs have reportedly developed in association with surgery, trauma, tumor and intracranial hemorrhage [2-6]. It was suggested that these conditions compromise the blood flow of dural sinuses, resulting in venous hypertension and cerebral ischemia which in turn initiate the formation of DAVF. Other clinical conditions that are rarely associated with DAVF include protein S deficiency, thrombophilia, antiphospholipid antibody syndrome and presence of factor $V$ Leiden or prothrombin G20210A mutation [7-11]. Most of these inherited conditions lead to venous thromboembolism, further raising the possibility of CVST playing a significant role in the development of DAVF.

A close association between CVST and DAVF has been established. Tsai and colleagues reported that CVSTs were detected in 39\% patients with DAVFs, and all CVSTs were located within the sinuses around the DAVFs or the downstream venous outflow pathways [12]. Houser et al first described two patients with angiographic evidence of sigmoid and transverse sinus occlusion before the appearance of DAVF in the transverse sinuses [13]. Chaudhary et al re- 


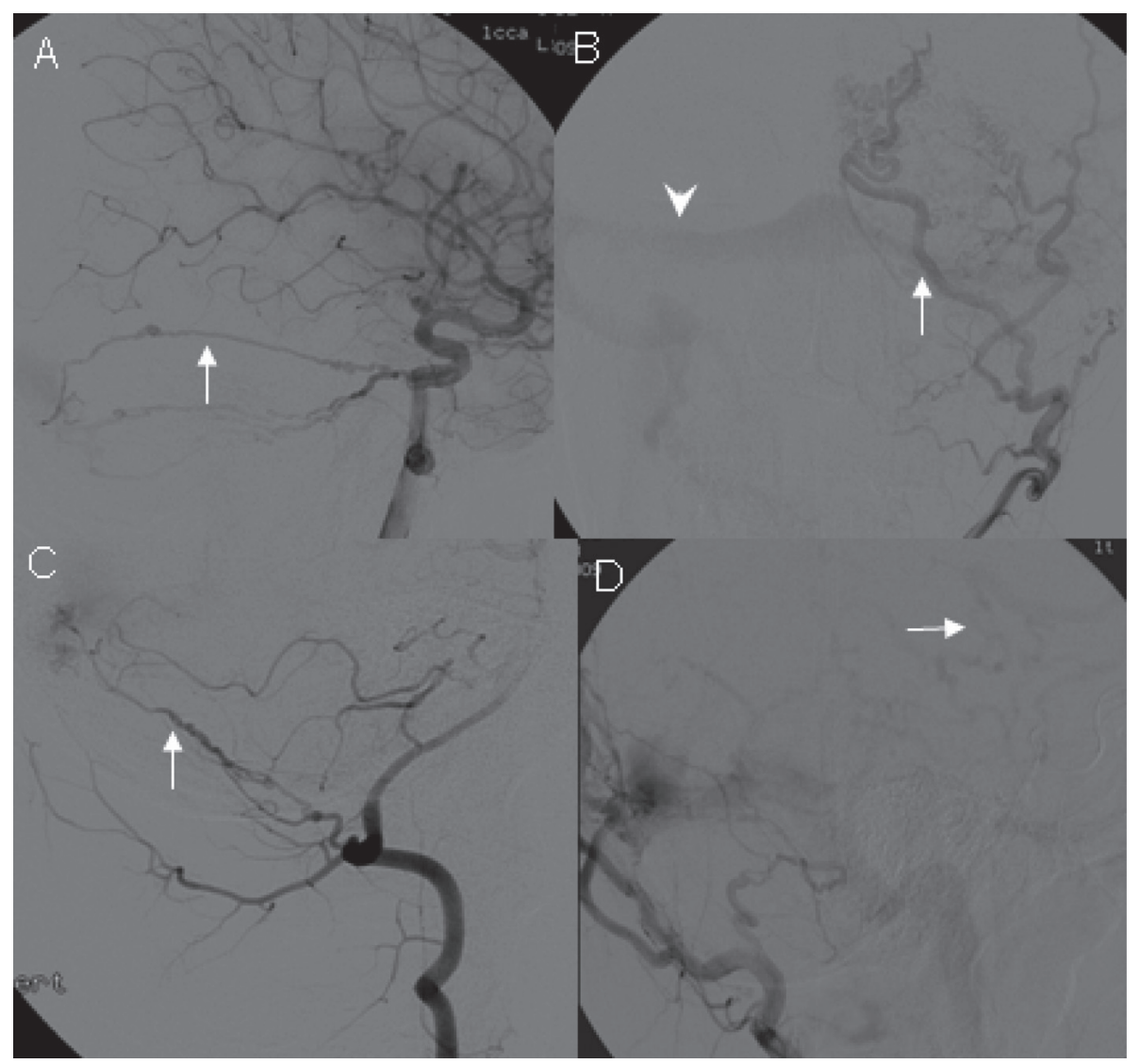

Figure 2. Digital subtractive angiography. At 14 months, a diagnostic arteriogram demonstrated a DAVF with feeders from the left internal carotid artery ( $A$, arrow indicating the tentorial branch), left external carotid artery (B, arrow indicating occipital branch; arrowhead indicating the patent right transverse sinus), and left vertebral artery ( $C$, arrow indicating the meningeal branch). Prominent cortical venous reflux was also observed ( $D$, arrow).

ported two cases of transverse and sigmoid sinus thrombosis prior to the development of DAVF [2]. DAVF can appear as early as three months after the formation of CVST [14]. Histological examination of surgically resected sinuses in patients with DAVF revealed subintimal fibrous thickening and hypertrophic changes in the sinus wall, suggesting a possible existence of previous CVST [15]. Conversely, CVST may emerge in the course of DAVF because of the stenosis or occlusion of sinus lumen. Among the 5 cases demonstrating the coexistence of DAVF and CVST in one report, DAVF was probably the causative factor in 1 patient leading to CVST formation [16]. Nishijima and his colleagues made similar observations of DAVF being the primary lesion in causing CVST [17]. Therefore, a mutually promoting relationship could exist between CVST and DAVF, and it may be difficult in some cases to ascertain which of the two is exactly the primary event.

Two hypotheses were proposed to account for the development of DAVF following CVST. The existing shunt theory states that an increase in the sinus and venous pressure by CVST may open the dormant arteriovenous shunts between meningeal arteries and dural venous sinuses. The angiogenesis theory proposed that neovascularization or angiogenesis initiates as a response to CVST and venous hypertension. Based on observations from murine DAVF models, Lawton et al suggested that venous hypertension leads to a reduced cerebral perfusion which promotes aberrant angiogenesis [18]. The formation of DAVF further intensifies the venous hypertension and causes a vicious cycle. Consistent with the angiogenesis theory, a strong expression of angiogenic growth factors has been identified in the human DAVF tissue [19].

Here we report a case of de novo DAVF formation following diffuse CVSTs in an asymptomatic patient. We believe that the presence of CVST was the cause for the subsequent development of DAVF in our patient based on both the temporal and spatial relationship. The long delay between the initial CVST and subsequent DAVF formation in our case is consistent with the angiogenesis theory rather than the existing shunt hypothesis. Diffuse cortical vein involvement was evident on multiple images in our patient (Fig. 2). Endovascular embolization procedures were adopted for this 
case due to the concerns for future complications such as hemorrhage, brain edema, stroke, seizures or dementia [20].

CVST usually occurs in young and healthy patients. The most common sites of thrombosis are the transverse and sigmoid sinuses. CVST is a rare disease with an estimated overall incidence of 5 people per million annually. However, a surprisingly higher prevalence of $9.3 \%$ was reported in a consecutive autopsy study suggesting that many patients were under-diagnosed and under-recognized [21]. CVST may present with signs and symptoms of intracranial hypertension or focal neurological deficits. Up to $25 \%$ of patients can be minimally symptomatic with nonspecific symptoms such as headache without focal neurological symptoms [22]. Head CT is widely used as the initial neuroimaging study in patients with new-onset neurological symptoms such as headache, seizure or mental status change. However, noncontrast head CT has a limited sensitivity of less than $30 \%$ for CVST [22]. Causes of CVST are numerous, nonspecific and obscure in most cases. All these features add to the difficulties in diagnosing CVST and a significant portion of cases could be easily missed. Our patient presented with a brief syncopal episode that was attributed to dehydration. At the initial presentation, there was a complete lack of focal neurological symptoms and signs despite the extensive CVST noted on imaging. To our knowledge, this is the first report describing an extensive CVST leading to DAVFs in a patient with essentially no attributable symptoms. Without brain imaging studies, CVST would have been overlooked in this case, and the subsequent development of DAVF would have been interpreted as being idiopathic or congenital.

Current practice guidelines recommend an initial usage of heparin or low molecular heparin, followed by warfarin for a period of 3 to 6 months for CVST patients with transient risk factors such as dehydration [22]. Our patient had no other identifiable risk factors of venous thrombosis. He received oral anticoagulation treatment for six weeks only. At the time when his anticoagulation treatment was discontinued, prominent venous blood flow was seen on the left side but the left transverse sinus remained narrow. At followup the left sigmoid sinus and left jugular vein were totally occluded, and DAVF formation on the left side was identified 14 months later on angiography. We suspect that a longer anticoagulation treatment could have changed his clinical course and might have avoided the subsequent DAVF development.

Our speculation is that in many DAVF cases, the diagnosis of previously-existing CVST has been missed. This is due to the inherent difficulties in recognizing CVST for the following reasons: the frequent absence of a unique etiology, significant neurological symptoms and the low sensitivity of common imaging techniques such as non-contrast CT. We do not suggest that all DAVF cases are associated with CVST, and it is also true that CVST does not always result in DAVF. Preter et al reported 77 CVST patients with a mean follow-up period of 6.5 years. Only $1(1.3 \%)$ patient developed DAVF 12 months later [23]. However, our case suggests that a high index of suspicion is required in studying the causal relationship between CVST and DAVF, and early recognition and appropriate treatment of CVST may lead to a successful prevention of DAVF in some cases.

\section{Declaration}

There are no conflicts of interest, financial or otherwise, associated with this submission.

\section{References}

1. Mironov A. Pathogenetical consideration of spontaneous dural arteriovenous fistulas (DAVFs). Acta Neurochir (Wien). 1994;131(1-2):45-58.

2. Chaudhary MY, Sachdev VP, Cho SH, Weitzner I, Jr., Puljic S, Huang YP. Dural arteriovenous malformation of the major venous sinuses: an acquired lesion. AJNR Am J Neuroradiol. 1982;3(1):13-19.

3. Awad IA, Little JR, Akarawi WP, Ahl J. Intracranial dural arteriovenous malformations: factors predisposing to an aggressive neurological course. J Neurosurg. 1990;72(6):839-850.

4. Ishikawa T, Houkin K, Tokuda K, Kawaguchi S, Kashiwaba T. Development of anterior cranial fossa dural arteriovenous malformation following head trauma. Case report. J Neurosurg. 1997;86(2):291-293.

5. Arnautovic KI, Al-Mefty O, Angtuaco E, Phares LJ. Dural arteriovenous malformations of the transverse/ sigmoid sinus acquired from dominant sinus occlusion by a tumor: report of two cases. Neurosurgery. 1998;42(2):383-388.

6. Desal HA, Lee SK, Kim BS, Raoul S, Tymianski M, TerBrugge KG. Multiple de novo vascular malformations in relation to diffuse venous occlusive disease: a case report. Neuroradiology. 2005;47(1):38-42.

7. Witt O, Pereira PL, Tillmann W. Severe cerebral venous sinus thrombosis and dural arteriovenous fistula in an infant with protein S deficiency. Childs Nerv Syst. 1999;15(2-3):128-130.

8. Kraus JA, Stuper BK, Nahser HC, Klockgether T, Berlit P. Significantly increased prevalence of factor $\mathrm{V}$ Leiden in patients with dural arteriovenous fistulas. J Neurol. 2000;247(7):521-523.

9. Safavi-Abbasi S, Di Rocco F, Nakaji P, Feigl GC, Gharabaghi A, Samii M, Valavanis A, et al. Thrombophilia Due to Factor V and Factor II Mutations and Formation of a Dural Arteriovenous Fistula: Case Report and Review of a Rare Entity. Skull Base. 2008;18(2):135-143.

10. van Dijk JM, TerBrugge KG, Van der Meer FJ, Wal- 
lace MC, Rosendaal FR. Thrombophilic factors and the formation of dural arteriovenous fistulas. J Neurosurg. 2007;107(1):56-59.

11. Miki Y, Tomiyama M, Arai A, Kimura T, Suzuki C, Nunomura J, Midorikawa $\mathrm{H}$, et al. Cerebral venous thrombosis with dural arteriovenous fistulas and antiphospholipid syndrome: a case report. Neurol Sci. 2010;31(2):237-238.

12. Tsai LK, Jeng JS, Liu HM, Wang HJ, Yip PK. Intracranial dural arteriovenous fistulas with or without cerebral sinus thrombosis: analysis of 69 patients. J Neurol Neurosurg Psychiatry. 2004;75(11):1639-1641.

13. Houser OW, Campbell JK, Campbell RJ, Sundt TM, Jr. Arteriovenous malformation affecting the transverse dural venous sinus--an acquired lesion. Mayo Clin Proc. 1979;54(10):651-661.

14. Morales H, Jones BV, Leach JL, Abruzzo TA. Documented development of a dural arteriovenous fistula in an infant subsequent to sinus thrombosis: case report and review of the literature. Neuroradiology. 2010;52(3):225-229.

15. Sakaki T, Morimoto T, Nakase H, Kakizaki T, Nagata $\mathrm{K}$. Dural arteriovenous fistula of the posterior fossa developing after surgical occlusion of the sigmoid sinus. Report of five cases. J Neurosurg. 1996;84(1):113-118.

16. Pierot L, Chiras J, Duyckaerts C, Jason M, Martin N. Intracranial dural arteriovenous fistulas and sinus throm- bosis. Report of five cases. J Neuroradiol. 1993;20(1):918.

17. Nishijima M, Takaku A, Endo S, Kuwayama N, Koizumi F, Sato H, Owada K. Etiological evaluation of dural arteriovenous malformations of the lateral and sigmoid sinuses based on histopathological examinations. J Neurosurg. 1992;76(4):600-606.

18. Lawton MT, Jacobowitz R, Spetzler RF. Redefined role of angiogenesis in the pathogenesis of dural arteriovenous malformations. J Neurosurg. 1997;87(2):267-274.

19. Uranishi R, Nakase H, Sakaki T. Expression of angiogenic growth factors in dural arteriovenous fistula. J Neurosurg. 1999;91(5):781-786.

20. Wilson M, Enevoldson P, Menezes B. Intracranial dural arterio-venous fistula. Pract Neurol. 2008;8(6):362-369.

21. Towbin A. The syndrome of latent cerebral venous thrombosis: its frequency and relation to age and congestive heart failure. Stroke. 1973;4(3):419-430.

22. Saposnik G, Barinagarrementeria F, Brown RD, Jr., Bushnell CD, Cucchiara B, Cushman M, deVeber G, et al. Diagnosis and management of cerebral venous thrombosis: a statement for healthcare professionals from the American Heart Association/American Stroke Association. Stroke. 2011;42(4):1158-1192.

23. Preter M, Tzourio C, Ameri A, Bousser MG. Long-term prognosis in cerebral venous thrombosis. Follow-up of 77 patients. Stroke. 1996;27(2):243-246. 